\title{
The Dynamic Structure of Regioregulated Poly(4-methylthiazole-2,5-diyl)
}

\author{
By Seiichiro MorI, ${ }^{1}$ Takakazu YAMAMoto,${ }^{2}$ Naoki ASAKAWA,${ }^{3}$ Koji YAZAWA,${ }^{1, *}$ and Yoshio INOUE
}

\author{
KEY WORDS: Quasiordered Structure / $\pi$-Conjugated Polymers / Solid-state NMR /
}

Elementally excitation of polymeric condensed materials is one of the key issues in understanding of both static and dynamic properties of these materials. For example, the dynamic conformational disorder called "conformon" is one of them found in a crystal. ${ }^{1}$ Some semicrystalline polymers are turned out to form the quasiordered phase, which is the mesophase defined by a partially disordered crystalline phase. ${ }^{2}$ This phase is similar to liquid crystals in which the order parameter is between those of the amorphous phase and the perfect crystal. However there are only a few studies concerning quasiordered phases of $\pi$-conjugated polymers ${ }^{3-9}$ in contrast to active researches on small molecules. ${ }^{10-16}$

So far investigations about the dynamics of phases have been sparse, mainly because there are limited available experimental methodologies. NMR spectroscopy is one of promising methods to obtain information about molecular dynamics since one can perform noninversive experiments. ${ }^{17-20}$ By various measuring techniques, dynamic properties can be observed over the wide range of frequencies from picoseconds to minutes.

Recently, a regioregulated $\pi$-electron conjugated polyheterocyclic with five-membered ring, poly(4-alkylthiazole-2,5diyl) (P4RTz), has beeen synthesized via dehalogenative organometallic polycondensation. ${ }^{21-26}$ The regioregulated head-to-head (HH-)P4MeTz shows physical properties remarkably different from the other types of P4RTz. ${ }^{25}$ The X-ray diffraction study indicates that neighboring $\mathrm{HH}-\mathrm{P} 4 \mathrm{MeTz}$ molecules take a face-to-face packing and the thiazole rings take coplanar structures along the main chain, forming a highly extended $\pi$-conjugated network. ${ }^{27}$ One of the interesting structural features of $\mathrm{HH}-\mathrm{P} 4 \mathrm{MeTz}$ is the alternative layered structure (Figure 1); a layer constituted by highly dense methyl groups with two dimensional manner and a layer with the faceto-face $\pi$-stacking.

It is known that the optical properties of $\pi$-conjugated polymers are strongly coupled to the electronic structure. Moreover, a variety of molecular dynamics influence the characteristics of electronic properties. Tretiak et al. have demonstrated that the vibrational slow motions, such as phenylene ring flip, in poly( $p$-phenylene vinylene) origomers are related to the relaxation of photoexcitation in the excited state. ${ }^{28}$ Therefore, understanding of the dynamic structure in $\pi$ conjugated polymers is an important and fundamental problem for the development of optical and electronic devices.

In this article, the dynamics of the quasiordered structure of $\mathrm{HH}-\mathrm{P} 4 \mathrm{MeTz}$ is investigated by differential scanning calorimetry (DSC), variable temperature ${ }^{2} \mathrm{D}$ quadrupolar echo NMR, and ${ }^{2} \mathrm{D}$ two-dimensional exchange (2DEX) NMR.

\section{EXPERIMENTAL}

HH-P4MeTz $\left(M_{\mathrm{w}}=3200\right.$; light scattering) was prepared by the previously reported schemes involving dehalogenative organometallic polycondensation. ${ }^{22}$ A methyl-deuterated version of HH-P4MeTz was synthesized from a scheme initiated from monobromination of deuterated acetone. The detailed scheme for the synthesis will be described in ref 22. For all the measurements, we used powder samples of HH-P4MeTz, which were recrystallized from hexafluoroisopropanol (HFIP) solution.

DSC thermograms of natural abundant and perdeuterated versions of HH-P4MeTz were recorded on a DSC220 system connected to a SSC5300 workstation (Seiko Instruments. Co. Ltd., Tokyo, Japan). The samples were heated from 173 to $423-473 \mathrm{~K}$ at a heating rate of 5 and $20^{\circ} \mathrm{C} / \mathrm{min}$.

A standard quadrupolar echo pulse sequence was used for ${ }^{2} \mathrm{D}$ NMR measurements. ${ }^{29}$ The rf intensity was set at $64 \mathrm{kHz}$. The recycle delay for all the experiments was $30 \mathrm{~s}$. Signal average was performed with 32-256 scans. The echo time for quadrupolar echo measurements was set at $0.020 \mathrm{~s}$.

For investigation of dynamic processes, such as chemical exchange, cross relaxation, spin diffusion, and atomic hopping motion, the 2DEX NMR has a number of advantages over the one-dimensional techniques. The 2DEX NMR method provides particularly valuable information on slow dynamics in solids. ${ }^{30-34}$ Dynamics, such as diffusion and/or reorientation, of certain atom (or molecule) is detected in the form of frequency change during the mixing time, $t_{\text {mix }}$. Moving nuclei

\footnotetext{
${ }^{1}$ Department of Biomolecular Engineering, Tokyo Institute of Technology, 4259-B55 Nagatsuta-cho, Midori-ku, Yokohama 226-8501, Japan

${ }^{2}$ Chemical Resources Laboratory, Tokyo Institute of Technology, 4259 Nagatsuta-cho, Midori-ku, Yokohama 226-8503, Japan

${ }^{3}$ Institute of Scientific and Industrial Research, Osaka University, 8-1 Mihogaoka 567-0047, Japan

*To whom correspondence should be addressed (Tel: +81-45-924-5796, E-mail: kyazawa@ titech.ac.jp).
} 


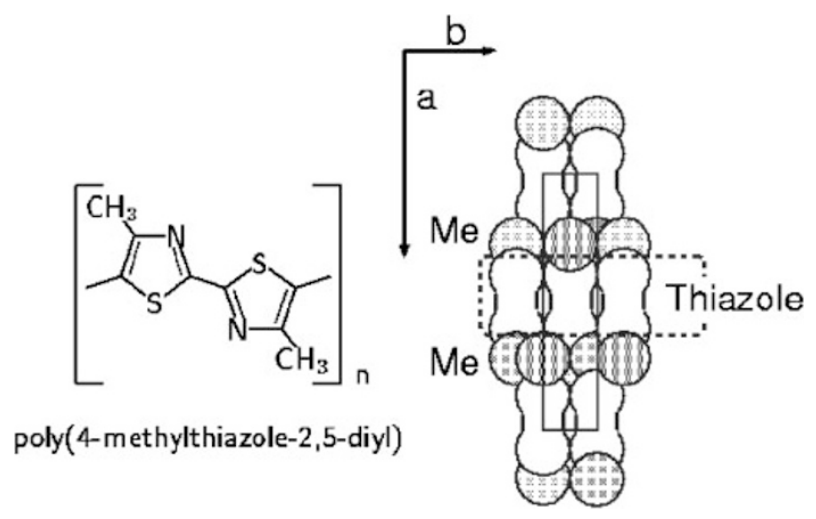

Figure 1. Chemical structure and schematic representation of the alternative layered molecular packing in $\mathrm{HH}-\mathrm{P} 4 \mathrm{MeTz}$. Thiazole rings take the intermolecular face-to-face packing along the $b$ axis and coplanar conformation along the $\mathrm{c}$ axis, which results in a highly extended $\pi$-conjugated network. Regioregulated $\mathrm{HH}-\mathrm{P} 4 \mathrm{MeTz}$ has the alternative layered structure: a layer constituted by densely-packed methyl groups with two dimensional manner and a layer with the face-to-face $\pi$-stacking.

experience a change of resonace frequency between at the beginning and at the end of $t_{\mathrm{mix}}$ and then create cross peaks, while static nuclei without changing the frequency (or moving nuclei with a frequency of much higher than $1 / t_{\text {mix }}$ ) produce the diagonal signals in the two-dimensional spectrum.

\section{RESULTS AND DISCUSSION}

The DSC thermograms (Figure 2) show an endo-thermal peak with a specific heat jump near $300 \mathrm{~K}$ for both natural abundant and perdeuterated samples. For perdeuterated $\mathrm{HH}-$ $\mathrm{P} 4 \mathrm{MeTz}$, the endo-themal peak at a heating rate of $20^{\circ} \mathrm{C} / \mathrm{min}$ was larger than that at $5^{\circ} \mathrm{C} / \mathrm{min}$ but both peaks began near $300 \mathrm{~K}$ regardless of the heating rate. It is well-known that a general $\pi$-conjugated polymer does not have a melting peak but a heat-resistant. It is difficult to consider this transition as a glass transition because for these samples there is no heating rate dependency of beginning temperature of the transition. Moreover, the possibility of recrystallization can be ruled out because the change of heat of fusion is opposite to that brought

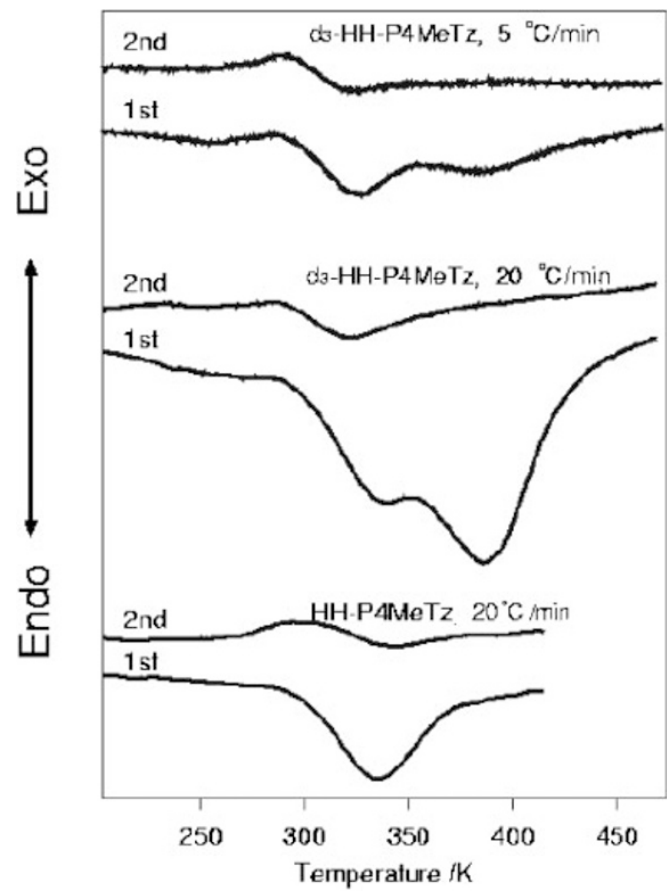

Figure 2. DSC charts for powder samples of perdeuterated $\mathrm{HH}-\mathrm{P} 4 \mathrm{MeTz}$ (top and middle) and natural abundant HH-P4MeTz (bottom). The heating rate is shown in the figure. After the first heating scans, the samples were rapidly quenched with liquid nitrogen and then they were again heated at the same rate as the first scans.

by the recrystallization. With taking the peculiar and rigid packing structure of $\mathrm{HH}-\mathrm{P} 4 \mathrm{MeTz}$ (Figure 1) into account, we suppose that this transition is a kind of structural phase transition, an "order-disorder" phase transition attributed to the gradual melting of intermolecular face-to-face $\pi$-stacking. ${ }^{35}$

In the ${ }^{2} \mathrm{D}$ quadrupolar NMR spectra, an axially symmetric character was observed in the quadrupolar powder spectra for the methyl deuterium atom (Figure 3). At higher temperatures, a sharp center peak due to melting was not observed, while the perpendicular edge showed a slight change attributed to an another quadrupolar coupling tensor. These results can be explained by the effect of wagging motion. ${ }^{36}$ Moreover, signal (a)

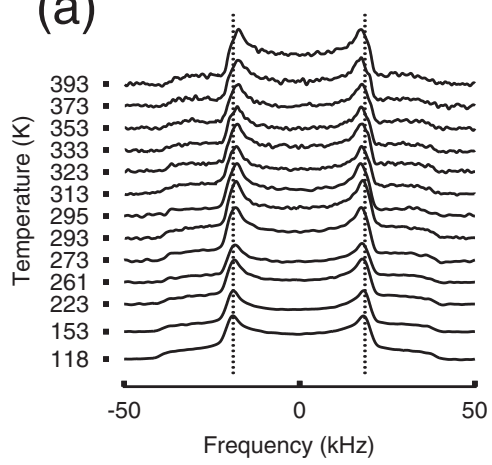

(b)

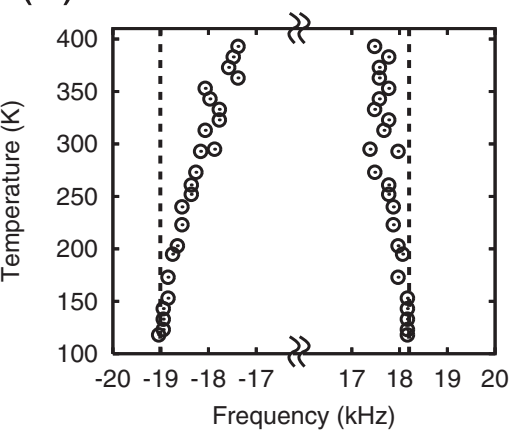

Figure 3. (a) $41 \mathrm{MHz}^{2} \mathrm{D}$ quadrupolar echo NMR spectra for the powdered sample of perdeuterated HH-P4MeTz at various temperatures (118-393 K). (b) The frequency change of the perpendicular edges plotted as a function of temperature. The dashed line is only a guide for eyes. 
$\mathrm{T}(\mathrm{K})$

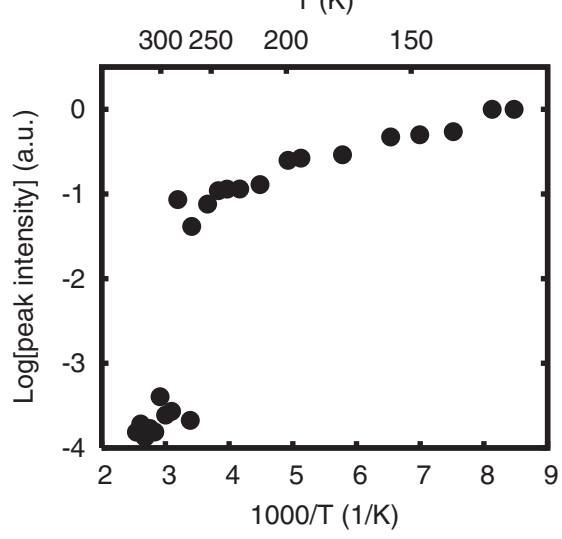

Figure 4. The semilogarithmic plot of normalized peak intensity as a function of temperature. Each peak intensity is converted into the one per a scan and normalized by the intensity at the highest temperature (393K).

intensities of the spectra drop drastically near $300 \mathrm{~K}$, indicating an appearance of molecular motion (Figure 4). Therefore, it is suggested that the wagging motion of methyl groups triggered by the partial melting of $\pi$-stacking will occur and the quasiordered phase is formed near $300 \mathrm{~K}$.

In order to explore more detailed molecular dynamics, 2DEX NMR measurements were performed. Figure 5 shows the 2DEX NMR spectra for perdeuterated HH-P4MeTz at 288 and $333 \mathrm{~K}$ observed as a function of mixing time $\left(t_{\text {mix }}\right)$. At $288 \mathrm{~K}$ (Figure 5(a), 5(b)), which is nearly the beginning of the phase transition inferred from the DSC results, the maximum frequency width $(\Delta \omega)$ increases with the increase of mixing time as listed in Table I, indicating the slow wagging motion of the methyl groups in the frequency scale of milliseconds. In addition, the intensity of the diagonal signals diminishes and disappears gradually with increase in mixing time, which means that most of the methyl groups may undergo the slow motion. The correlation time $\left(\tau_{\mathrm{ex}}\right)$ for the wagging motion can be determined from the following equation, ${ }^{33,34}$

$$
\Delta \omega=A \tanh \left(\frac{t_{\mathrm{mix}}}{\tau_{\mathrm{ex}}}\right),
$$

where the parameter $A$ corresponds to a saturation value of the curve. Using this equation, $\tau_{\mathrm{ex}}$ is found to be $6.7 \mathrm{~ms}(\sim 147 \mathrm{~Hz})$ at $288 \mathrm{~K}$. Since saturation of the frequency width $(\Delta \omega)$ is an evidence of the existence of spatially restricted molecular motion (Figure 6), it is suggested that in HH-P4MeTz the thiazole rings can not perform a fast rotation or flipping near $288 \mathrm{~K}$.

Figure 5(c) and 5(d) show 2DEX NMR spectra after heating the sample at $333 \mathrm{~K}$. For both mixing time no cross peaks was visible and actually the same spectrum was observed for all mixing time, indicateing the existence of rapid orientation due to the whole melting of $\pi$-stacking. When this sample was again cooled down to $288 \mathrm{~K}$, however, the result was not the same as that before heating, but the same as that after heating (Figure 5(e), 5(f)). From these spectra, we conjectured that in
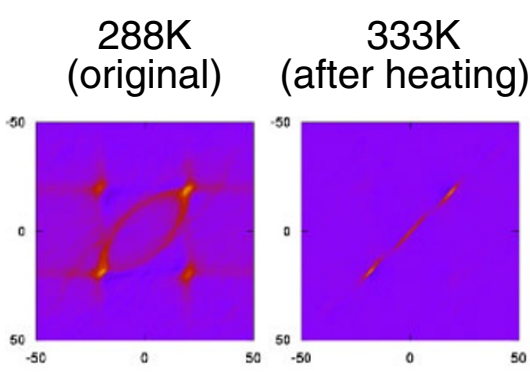

$288 \mathrm{~K}$

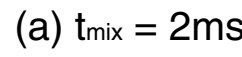

(c) $t_{\text {mix }}=2 \mathrm{~ms}$

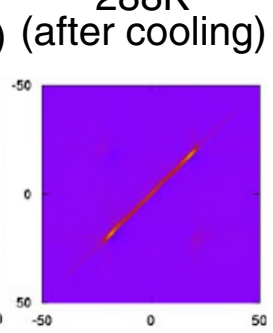

(e) $t_{\text {mix }}=2 \mathrm{~ms}$
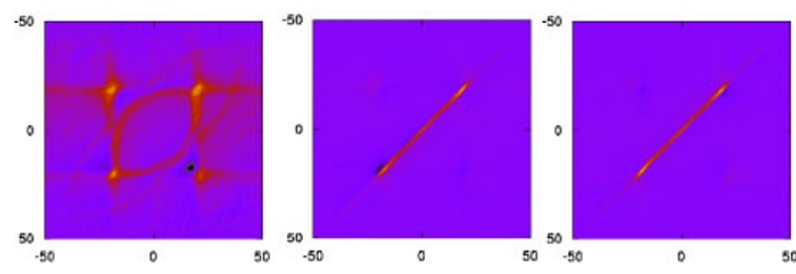

(b) $t_{\text {mix }}=3 \mathrm{~ms}$

(d) $t_{\text {mix }}=3 \mathrm{~ms}$

(f) $t_{\text {mix }}=3 \mathrm{~ms}$

Figure 5. ${ }^{2} \mathrm{D}$ 2DEX NMR spectra for perdeuterated $\mathrm{HH}-\mathrm{P} 4 \mathrm{MeTz}$ at 288 and $333 \mathrm{~K}$ with various mixing times. In the original sample, the cross peaks are observed and their intensities increase with mixing time $[(\mathrm{a}),(\mathrm{b})]$. After heating the sample to $333 \mathrm{~K}[(\mathrm{c}),(\mathrm{d})]$, only the diagonal peak was observed. The sample was again cooled down to $288 \mathrm{~K}[(\mathrm{e}),(\mathrm{f})]$. Tightly fixed or rapidly moving methyl groups will maintain intensities of the diagonal peaks, if they exist. In our measuring time scale, such the intensities were observed in the spectra after thermally treated sample.

Table I. The maximum frequency width observed by 2DEX NMR measurements with various mixing times at $288 \mathrm{~K}$

\begin{tabular}{cccccc}
\hline$t_{\text {mix }}(\mathrm{ms})$ & 0.5 & 1 & 2 & 3 & 5 \\
\hline$\Delta \omega(\mathrm{kHz})$ & 4.5 & 8.4 & 14.5 & 20.7 & 32.2 \\
\hline
\end{tabular}

the higher temperature range there exists rapid reorientation due to the whole melting of $\pi$-stacking and such motion does not freeze even at the lower temperature $(288 \mathrm{~K})$. Therefore, the rigid packing structure, which is necessary for the slow reorientation near the phase transition temperature, cannot be reproduced. This longtime and irreversible character might expect the use of HH-P4MeTz as the storage medium. These results show that the slow wagging motion of methyl groups induced by only partially weakened face-to-face $\pi$-stacking is the characteristic dynamics in the quasiordered phase of $\mathrm{HH}-$ P4MeTz.

In conclusion, the solid-state NMR spectroscopy is found to be a powerful method to investigate solid-state dynamics of regioregulated $\mathrm{HH}-\mathrm{P} 4 \mathrm{MeTz}$. DSC measurements show that both natural abundant and perdeuterated $\mathrm{HH}-\mathrm{P} 4 \mathrm{MeTz}$ undergo a phase transition near $300 \mathrm{~K}$. The ${ }^{2} \mathrm{D}$ quadrupolar NMR spectra observed at various temperatures strongly confirm the DSC observatoion that a phase transition from the crystalline to the quasiordered phase occurs in regioregulated HH-P4MeTz. The 2DEX NMR experiments revealed that the motion of the methyl groups on the order of milliseconds exists in $\mathrm{HH}$ $\mathrm{P} 4 \mathrm{MeTz}$ and the exchange rate of this motion was estimated to be $\tau_{\mathrm{ex}}{ }^{-1}=147 \mathrm{~Hz}$. 


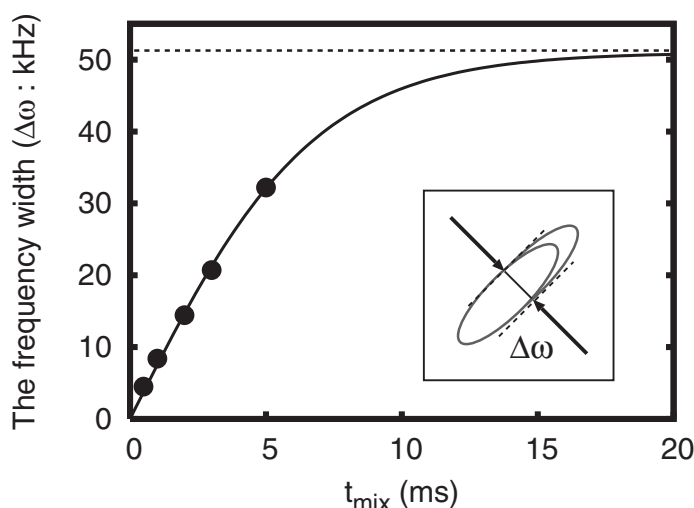

Figure 6. The variation of the frequency width $(\Delta \omega)$ with mixing time at $288 \mathrm{~K}$. The dotted straight line is an asymptotic value $(A=51 \mathrm{kHz})$ that the fitting curve reaches. From this fitting, $\boldsymbol{\tau}_{\mathrm{ex}}$ is estimated as $6.7 \mathrm{~ms}$

This research is an example of NMR measuring phonon dispersion. Such dynamics may be detectable due to the existence of distinctive one-dimensional fluctuation in the $\pi$ conjugated systems. It is highly likely that the NMR measurement is useful method for the research and development of electronical materials with low-dimensionality.

Received: November 13, 2007

Accepted: February 3, 2008

Published: March 19, 2008

\section{REFERENCES}

1. E. R. Andrew, Phys. Lett., 34A, 30 (1971).

2. C. Yang, F. P. Orfino, and S. Holdcroft, Macromolecules, 29, 6510 (1996).

3. J. W. van der Horst, P. A. Bobbert, and M. A. J. Michels, Phys. Rev. $B, 64,035206$ (2002).

4. K. Holczer, J. P. Boucher, F. Devreux, and M. Nechtschein, Phys. Rev. B, 23, 1051 (1981).

5. M. Nechtschein, F. Devreux, F. Genoud, M. Guglielmi, and K. Holczer, Phys. Rev. B, 27, 61 (1983).

6. B. R. Weinberger, E. Ehrenfreund, A. Pron, A. J. Heeger, and A. G. MacDiarmid, J. Chem. Phys., 72, 4749 (1980).

7. M. Nechtschein, F. Devreux, R. L. Greene, T. C. Clarke, and G. B. Street, Phys. Rev. Lett., 44, 356 (1980).

8. W. P. Su, J. R. Schrieffer, and A. J. Heeger, Phys. Rev. Lett., 42, 1698 (1979).
9. W. P. Su, J. R. Schrieffer, and A. J. Heeger, Phys. Rev. B, 22, 2099 (1980).

10. S.-B. Liu and M. S. Conradi, Phys. Rev. Lett., 54, 1287 (1985).

11. J. Etrillard, B. Toudic, H. Cailleau, and G. Coddens, Phys. Rev. B, 51, 8753 (1995).

12. L. von Laue, F. Ermark, A. Gölzhäuser, U. Haeberlent, and U. Häcker, J. Phys., Condens. Matter, 8, 3977 (1996).

13. K. Kohda, N. Nakamura, and H. Chihara, J. Phys. Soc. Jpn., 51, 3936 (1982).

14. J. O. Williams, Chem. Phys. Lett., 42, 171 (1976).

15. R. Blinc, U. Mikac, T. Apih, J. Dolinšek, J. Seliger, J. Slak, S. Žumer, L. Guibe, and D. C. Ailion, Phys. Rev. Lett., 88, 015701 (2002).

16. R. E. de Souza, M. Engelsberg, and D. J. Pusiol, Phys. Rev. Lett., 66, 1505 (1991).

17. W. L. Mcmillan, Phys. Rev. B, 14, 1496 (1976).

18. K. S. Novoselov, A. K. Geim, S. V. Dubonos, E. W. Hill, and I. V. Grigorieva, Nature, 426, 812 (2003).

19. T. Janssen and O. Radulescu, Z. Phys. B, 104, 657 (1997).

20. R. Blinc, D. C. Ailion, J. Dolinsek, and S. Zumer, Phys. Rev. Lett., 54, 79 (1985).

21. T. Yamamoto, H. Suganuma, T. Maruyama, and K. Kubota, J. Chem. Soc. Chem. Commun., 1613 (1995).

22. T. Maruyama, H. Suganuma, and T. Yamamoto, Synth. Met., 74, 183 (1995).

23. T. Yamamoto, Chem. Lett., 703 (1996).

24. T. Yamamoto, H. Suganuma, Y. Saitoh, T. Maruyama, and T. Inoue, Jpn. J. Appl. Phys., 35, L1142 (1996).

25. T. Yamamoto, D. Komarudin, M. Arai, B.-L. Lee, H. Suganuma, N. Asakawa, Y. Inoue, K. Kubota, S. Sasaki, T. Fukuda, and H. Matsuda, J. Am. Chem. Soc., 120, 2047 (1998).

26. T. Yamamoto, H. Suganuma, T. Maruyama, T. Inoue, Y. Muramatsu, M. Arai, D. Komarudin, N. Ooba, S. Tomaru, S. Sasaki, and K Kubota, Chem. Mater., 9, 1217 (1997).

27. T. Yamamoto, B.-L. Lee, H. Suganuma, and S. Sasaki, Polym. J., 30, 853 (1998).

28. S. Tretiak, A. Saxena, R. L. Martin, and A. R. Bishop, Phys. Rev. Lett., 89, 097402 (2002).

29. C. P. Slichter, "Principles of Magnetic Resonance", Springer-Verlag, Berlin, Heidelberg, 1978.

30. L. Muntean and D. C. Ailion, Phys. Rev. B, 62, 11351 (2000).

31. L. Muntean and D. C. Ailion, Phys. Rev. B, 63, 012406 (2000).

32. R. R. Ernst, G. Bodenhausen, and A. Wokaun, "Principles of Nuclear Magnetic Resonance in One and Two Dimensions”, Clarendon Press, Oxford, 1987.

33. J. Dolinšek, B. Ambrosini, P. Vonlanthen, J. L. Gavilano, M. A. Chernikov, and H. R. Ott, Phys. Rev. Lett., 81, 3671 (1998).

34. K. Schmidt-Rohr and H. W. Spiess, "Multidimensional Solid-state NMR and Polymers", Academic press, London, 1994.

35. L. G. Ronda, D. C. Martin, J. I. Nanos, J. K. Politis, and M. D. Curtis, Macromolecules, 32, 4558 (1999).

36. A. Kintanar, T. M. Alam, W. C. Haung, C. Schindele, D. E. Wemmer, and G. Drobny, J. Am. Chem. Soc., 110, 6367 (1988). 